\title{
Forest vegetation Interaction with L-band Satellite Signals
}

\author{
Vladimir Podoprigora ${ }^{1,2}$, and Anatoly Sorokin ${ }^{2,3}$ \\ ${ }^{1}$ Siberian Federal University, 79 Svobodny av., 660041 Krasnoyarsk, Russia. \\ ${ }^{2}$ Federal Research Center, 50 Akademgorodok, 6660036 Krasnoyarsk, Russia \\ ${ }^{3}$ Siberian University of Science and Technology, 31 Krasnoyarskij Rabochij av., 660037 \\ Krasnoyarsk, Russia
}

\begin{abstract}
A method for calculating the electro-physical parameters of forest vegetation cover interacting with the signals of navigation satellites in the frequency bands $1.2-1.6 \mathrm{GHz}$ is proposed. A technique for the experimental measurement of linear attenuation coefficient of satellite signals on test areas of canopies was developed, taking into account their calibration at the input. The proposed forest vegetation model describes the group of crowns as a system of quasi spherical "capsules", located chaotically in the air matrix. The scattering and penetration cross sections and the coefficient of linear attenuation of radio waves were calculated using the approximation of anomalous diffraction for crowns and the results of solving the diffraction problem for trunks. The effects of multiple scattering were taken into account.
\end{abstract}

\section{Introduction}

Currently, the Earth's forest vegetation cover monitoring using modern technologies in remote sensing over high frequency (UHF) radio waves becomes more actual. To determine the state of forested area using remote radio wave satellite sounding, it is necessary to solve the inverse problem based on the finding functional and statistical dependencies between the electro physical parameters of the forest and the received satellite signals. However, it is possible to found the relation between biometric and taxation characteristics of the forest vegetation cover and parameters of the processed signals only based on the corresponding electro physical models of the forest vegetation.

\section{Model of the interaction the L-band signals with the forest}

To describe the propagation of radio emission in the forest, the models [1] are used. They represent the vegetation cover either as a continuous dielectric medium with random or as a discrete set of elements. The application a particular model is specified by the conditions of small dimensions of the forest elements (leaves, needles, branches, trunks) in comparison with the wavelength of the incoming radiation. In the microwave range of satellite signals, the dimensions of forest elements are commensurable with the wavelength $\lambda$ of an electromagnetic wave ( $\lambda=1-30 \mathrm{~cm}$ ), so the calculation of scattering, penetration and attenuation cross sections should be based on diffraction models that take into account the shape and dimensions of the elements. Rigorous solution of the diffraction problem for 
disks, strips (leaves), cylinders (needles, trunks, branches) does not exist. Therefore, these sections can be found only under certain restrictions. There are not enough works analyzing the relation between satellite radiofrequencies in the L-band $(1.2-1.6 \mathrm{GHz})$ and the forest area, moreover their conclusions can be ambiguous.

The present study is devoted to the development of techniques and experimental measurement of the signals of GLONASS and GPS satellites interacting with forest vegetation coverage of various types, as well as methods for calculating the basic parameters of this interaction: the coefficient of linear attenuation, scattering and penetration cross sections.

Three areas of the Krasnoyarsk Academgorodok with an area of $\approx 900-1000 \mathrm{~m}^{2}$ each were used as the experimental site was used by there. It were covered with pine and birch trees with different thicknesses, without undergrowth. The age of trees was 60-70 years. The average size parameters are given in Table 1.

Table 1. Forest stand parameters

\begin{tabular}{|c|c|c|c|c|}
\hline $\begin{array}{c}\text { Forest } \\
\text { type }\end{array}$ & $\begin{array}{c}\text { Height of } \\
\text { trees, } \mathrm{m}\end{array}$ & $\begin{array}{c}\text { Crown } \\
\text { height, } \mathrm{m}\end{array}$ & $\begin{array}{c}\text { Diameter of the } \\
\text { trunk, } \mathrm{m}\end{array}$ & $\begin{array}{c}\text { Cross-sectional } \\
\text { area, } \mathrm{m}^{2}\end{array}$ \\
\hline Pine & 23 & 7 & 0.31 & 0.075 \\
\hline Birch & 21 & 8 & 0.32 & 0.080 \\
\hline
\end{tabular}

The average distance between trees for a dense tree stand was $3.5 \mathrm{~m}$. The density of trees was 0.11 trunks $/ \mathrm{m}^{2}$ (1100 trees per 1 hectare), the average distance between the pines for broken forest was $9.9 \mathrm{~m}$ with the density 0.05 trunks $/ \mathrm{m}^{2}$. The average distance between the birches was $8.2 \mathrm{~m}$, the density was 0.06 trunks $/ \mathrm{m}^{2}(600$ trees per 1 hectare).

The effective dielectric constant at a frequency of $1.4 \mathrm{GHz}$ was chosen: for coniferous forest $\mathrm{E}=1.001+0.0002 \mathrm{i}$; for deciduous forest $\mathrm{E}=1.0028+0.0006 \mathrm{i}[2]$.

The processes of scattering, penetration and attenuation in the most general discrete model are described by the classical Rayleigh-Gans (RG) transformation. Point dipoles are often used for particles with dimensions much smaller than the length of an electromagnetic wave. Large scatterers are described by the anomalous diffraction (AD). Other models reviewed in [1], for example, the radiation transfer model [3], MIMIC [4], are also used.

This paper represents a forest model that describes a group of crowns as a system of quasispherical "capsules" with radius R. They are located randomly in the air matrix and have a different internal structure of the branches arrangement depending on the tree species. Since crowns in decimeter wave range have a value of $k R=2 \pi R / \lambda \gg 1$ at $\mathrm{R}=1.5-2 \mathrm{~m}$ and the relative refractive index of a crown is close to one, the $\mathrm{AD}$ approximation was used to calculate the scattering cross section.

In this approximation, assuming the crown of the tree to be a "capsule" with a radial arrangement of branches, we obtain we obtain total cross section the scattering $\sigma_{\mathrm{p}}$ for the one tree crown for the total cross section:

$$
\sigma_{p}=2 \sigma_{0} \int_{0}^{1}\left(2-\cos \Delta_{l l}-\cos \Delta_{l}\right) y d y .
$$

Here $\sigma_{0}$ is a geometric cross-sections of scattering, $\Delta_{I I}$ and $\Delta_{I}$ are quantities that depend on the effective permittivity of the tree crown, its radius $\mathrm{R}$ and the angle between the wave vector $\overline{\boldsymbol{k}}$ and the vertical line; y is a integration variable equal to the relation to the radius of scattering vector projection onto a plane orthogonal to the wave propagation vector [5].

The effects of multiple scattering on spherical tree crowns were taken into account in calculating the differential scattering cross-section $\left(\frac{d \sigma_{p}}{d \Omega}\right)_{N}$ by a system of $\mathrm{N}$ spheres, which can be written as 


$$
\left(\frac{d \sigma_{p}}{d \Omega}\right)_{N}=N\left\{\left(\frac{d \sigma_{p}}{d \Omega}\right)_{N}\left[1+N_{v} g\left(k_{p}, \eta, R\right)\right]\right\}=N \frac{d \sigma_{p}^{\ni \phi}}{d \Omega}
$$

where $\Omega$ is a solid angle of scattering, $N_{v}$ is an average density of spheres,

$$
g(k, \eta, R)=4 \pi \int_{0}^{\infty} G\left(r^{1}\right) \frac{\sin k_{p} r^{1}}{k_{p} r^{1}} r^{2} d r^{1}
$$

is the Fourier transform of the radial distribution function $G\left(r^{l}\right)$, which depends on the difference between the wave vectors of incoming and scattered waves $k_{p}=k-k^{l}$, a radius of the spheres $\mathrm{R}$ and the volume $\eta$ occupied by spheres of crowns, $\eta=N_{v}\left(\frac{4}{3} \pi R^{3}\right) ; \frac{d \sigma_{p}^{\ni \phi}}{d \Omega}$ is the effective differential scattering cross-section. The equation (2) consists of two parts: 1) the scattering differential cross-section of an isolated sphere multiplied by $N ; 2$ ) the correlation member $N_{v} g\left(k_{p}, \eta, R\right) \frac{d \sigma_{p}}{d \Omega} N$, which depends on the phase difference between the scattered fields and the position of the spheres in the ensemble.

The most realistic way of taking into account the correlation effects in scattering is the correlation function used in [6] and suitable for describing media with high concentration of particles.

The dependences $d \sigma_{p} / d \Omega$ on the scattering angle calculated from formula (2) at different tree concentrations $\eta$ show that the effects of multiple radio waves scattering on crowns are noticeably manifested at small observation angles. The influence of crowns concentration on the degree of scattering medium homogeneity can be characterized by the ratio $\sigma_{p}^{\ni \phi} / \sigma_{p}$.

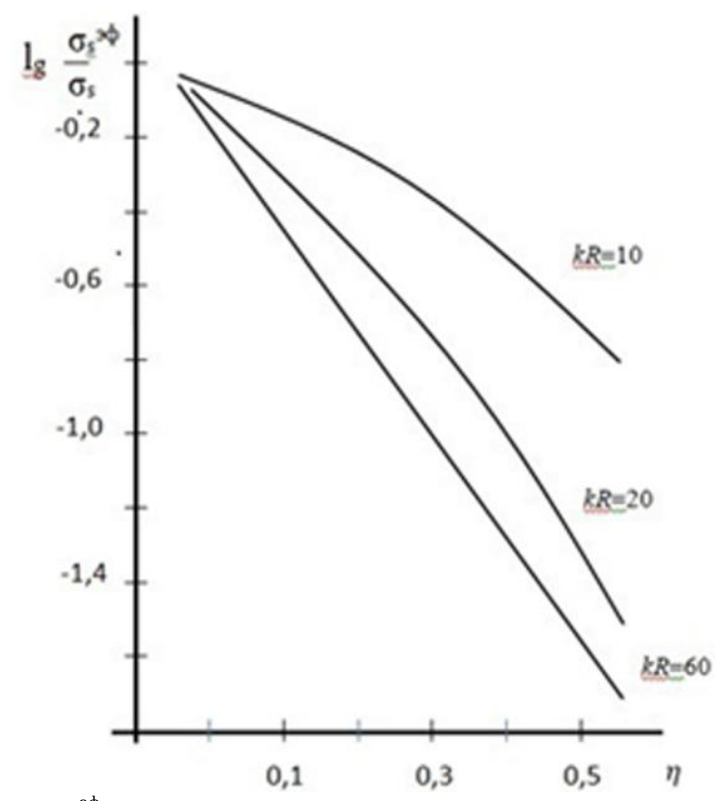

Fig.1. Dependencies of $\log \frac{\sigma_{p}^{3 \phi}}{\sigma_{p}}$ on the volume concentration of crowns $\eta$ for different values of $k R$.

It can be seen from Fig. 1., that as the concentration increases, the effective scattering cross-section $\sigma_{p}^{\ni \phi}$ decreases in comparison with $\sigma_{p}$.

The different slope of the curves $f(\eta)=\frac{\sigma_{p}^{э \phi}}{\sigma_{p}}$ indicates that the degree of homogeneity depends on the size of the spheres. The radiation transfer equations showed that at small 
concentration of particles in the medium $\sigma_{p}^{\ni \phi}$ is equal to the scattering cross-section of a particular particle in the matrix. When concentration increases, a value of $\sigma_{p}^{\ni \phi}$ decreases, and at $\sigma_{p}^{\ni \phi} \ll \sigma_{p}$ the "crown-air" scattering medium can be considered as quasi homogeneous, therewith the radio wave propagation in it can be described as in the case of continuous homogeneous medium.

The attenuation and the scattering cross-sections [7, 8] at the trunks and branches were calculated using the equations derived from the solution to the diffraction problem for infinite circular cylinders. They was presented as series containing the functions of Bessel and Hankel. The calculation of the cross-sections $\sigma_{0}$ and $\sigma_{p}$ shows that the effect of resonance interaction of $\mathrm{L}$ - band radio waves with trunks and branches is significant. The group of the trunks are presented by a statistical ensemble characterized by a normal distribution of tree parameters eight, diameter and distance between them). The average orientation of the thick branches, contributing to the scattering cross-section, is described by the "order parameter", determined by the slope angles cosines of the branches relative to the vertical.

\section{Conclusion}

The experimental studies were carried out using the method for determining the local coefficient of linear attenuation of $\gamma$ signals from navigation satellites in the forest developed in [9]. The values of $\gamma$, measured in a variation of electromagnetic transmission probing for pine and birch stands, are in satisfactory agreement with the calculated values of the linear attenuation coefficient. For example, for the GLONASS satellite system and the pine forest $\gamma_{\text {exp }}=-0,26 \pm 0,02 \mathrm{~dB} / \mathrm{m}, \gamma_{\text {calculated }}=-0,23 \mathrm{~dB} / \mathrm{m}$. This $/$ study allows us to hope for the applicability of the described approach for solving other problems of remote sensing of forest vegetation.

\section{References}

1. V.I. Popov, Propagation of radio waves in forests - M.: Hot line - Telecom,.- 392 p. (2015)

2. A.A. Milshin,A.G.Granov. Study of the Earth from Space.3, 50 (2000).

3. A.Ishimaru. Wave Pro pagation and Sca tt ering in Random Media (Academic Press, New York, 1978).

4. F. Ulaby, K.Sarabandy, Mc Donald Int. J. Remote Sensing. 11, 7, 1223 (1990).

5. S.ZumerPhys. Rev., 37, 10, 4006 (1988) .

6. H.Eyring, D. Henderson, B. Stover,Statis fecal Mechanies and Dynamics - Wiley, New York, 1982) .

7. A.Chukhlantsev, A. Shutko, S. Golovachev. Radio engineering and electronics, 48, 11, 1285 (2003).

8. S. Novik, A.Zavyalov, E. Telpuhovsky Proceedings of higher educational institutions. Physics. 3, 57 (2006).

9. A. Sorokin, V. Podoprigora, S. Fomin, I Savin. Proceedings of higher educational institutions. Physics. 60, 12, 19 (2017). 